\title{
PERIODIC SOLUTIONS OF ARBITRARY LENGTH IN A SIMPLE INTEGER ITERATION
}

\author{
DEAN CLARK
}

Received 28 May 2005; Accepted 19 July 2005

We prove that all solutions to the nonlinear second-order difference equation in integers $y_{n+1}=\left\lceil a y_{n}\right\rceil-y_{n-1},\{a \in \mathbb{R}:|a|<2, a \neq 0, \pm 1\}, y_{0}, y_{1} \in \mathbb{Z}$, are periodic. The first-order system representation of this equation is shown to have self-similar and chaotic solutions in the integer plane.

Copyright (C) 2006 Dean Clark. This is an open access article distributed under the Creative Commons Attribution License, which permits unrestricted use, distribution, and reproduction in any medium, provided the original work is properly cited.

\section{Introduction}

We study the nonlinear second-order difference equation in integers

$$
y_{n+1}=\left\lceil a y_{n}\right\rceil-y_{n-1}, \quad\{a \in \mathbb{R}:|a|<2, a \neq 0, \pm 1\}, y_{0}, y_{1} \in \mathbb{Z},
$$

where $\lceil x\rceil$ denotes the smallest integer not smaller than $x$ (the ceiling function). The reader is already familiar with the linear cases $a=0, \pm 1$, therefore we do not consider these values in this paper. Besides the natural generalization to discrete space, there are at least three reasons why $(1.1)$ is interesting.

First, when $a=3 / 2$, (1.1) becomes

$$
y_{n+1}= \begin{cases}\frac{3 y_{n}+1}{2}-y_{n-1} & \text { if } y_{n} \text { is odd } \\ 3\left(\frac{y_{n}}{2}\right)-y_{n-1} & \text { if } y_{n} \text { is even }\end{cases}
$$

a second-order variant of the notorious " $3 x+1$ iteration." So far as we know, the ultimate convergence to 1 of the $3 x+1$ iterates remains an unproven conjecture. In contrast, we will prove an ultimate recurrence property for (1.1) for all initial states $y_{0}, y_{1} \in \mathbb{Z}$ and parameter values $-2<a<2$. It is the initial state that is always recurrent. Moreover, solutions to (1.1) can exhibit periods of arbitrary length (Theorems 2.2, 3.2, below). 
Second, the method used to establish the periodicity of all solutions to (1.1) seems novel, elegant, and of potentially wider applicability. Without this method, we could not prove that solutions of the special case (1.2), above, were even bounded [1].

Third, (1.1) is converted to a first-order system in two variables using the mapping $T(x, y)=(y,\lceil a y\rceil-x)$. The simplicity of $T$ gives no hint of the startling complexity shown by scatter plots of some of the solutions. See Figures 4.1-4.4, below.

\section{Qualitative behavior of solutions}

Henceforth, all pairs $(x, y)$ denote points in the integer plane $\mathbb{Z}^{2}$, and the real parameter $a$ satisfies $|a|<2$. We obtain the aforementioned first-order system by letting

$$
T(x, y)=(y,\lceil a y\rceil-x), \quad X_{n}=\left(x_{n}, y_{n}\right)=T^{n}\left(x_{0}, y_{0}\right), \quad n=0,1,2, \ldots
$$

Remark 2.1. The sequence $\left(y_{n}\right)$ appearing as the second coordinate in each term of $\left(X_{n}\right)$ is the same sequence generated by (1.1) when $x_{0}=\left\lceil a y_{0}\right\rceil-y_{1}$.

A first glimpse of the rotational motion of solutions is obtained from the powers of $A=\left(\begin{array}{cc}0 & 1 \\ -1 & a\end{array}\right)$, the matrix underlying $T$ without the ceiling function. Because $|a|<2, A$ has complex eigenvalues. After diagonalizing $A$, we have

$$
A^{n}=\left(\begin{array}{cc}
\cos (n \theta)-\frac{a \sin (n \theta)}{\sqrt{4-a^{2}}} & \frac{2 \sin (n \theta)}{\sqrt{4-a^{2}}} \\
-\frac{2 \sin (n \theta)}{\sqrt{4-a^{2}}} & \cos (n \theta)+\frac{a \sin (n \theta)}{\sqrt{4-a^{2}}}
\end{array}\right), \quad \theta=\arccos \left(\frac{a}{2}\right)
$$

The significance of $\theta$ for the nonlinear equation (1.1) will become apparent later.

The identity $x^{2}+y^{2}-a x y=y^{2}+(a y-x)^{2}-a y(a y-x)$ supplies a family of invariant ellipses $E(x, y)=x^{2}+y^{2}-a x y$ for the linear equation. Figure 2.1 shows the ellipse $x^{2}+$ $y^{2}-(1 / 2) x y$ determined by $a=1 / 2$ and $\left(x_{0}, y_{0}\right)=(0,32)$, as well as the first six iterates of $T$ acting on $\left(x_{0}, y_{0}\right)=(0,32):(0,32),(32,16),(16,-24),(-24,-28),(-28,10)$, and $(10,33)$. All these points lie on the ellipse $x^{2}+y^{2}-(1 / 2) x y=1024$ because the ceiling function is inactive. The first odd $y_{n}$ requiring use of the ceiling is $y_{5}=33$, and we expect that $T(10,33)=(33,7)$ does not lie on this ellipse. Indeed, it does not: $33^{2}+7^{2}-1 / 2$. $33 \cdot 7=1022.5$.

The clockwise motion in $\mathbb{Z}^{2}$ of the iterates of $T$ is further clarified by the vector field drawn in Figure 2.2 with $a=1 / 3$. Each directed segment at $(x, y)$ has the form $T(x, y)-$ $(x, y)$ and thus points toward $T(x, y)$. This is seen clearly in Figure 2.2 for the orbit initiated at $(1,0)$. The vector field is divided into four quadrants with boundaries $y=x$ and the step locus $y=\lceil a y\rceil-x$. Each linear segment of this locus includes the upper lefthand endpoint and excludes the lower right-hand endpoint. The quadrants discriminate whether direction vectors have $\Delta x>0(\leq 0)$ or $\Delta y>0(\leq 0)$. In general, the clockwise rotation and roughly elliptic orbits are easily confirmed for all parameter values $-2<a<2$ and initial conditions $\left(x_{0}, y_{0}\right) \neq T\left(x_{0}, y_{0}\right)$. 


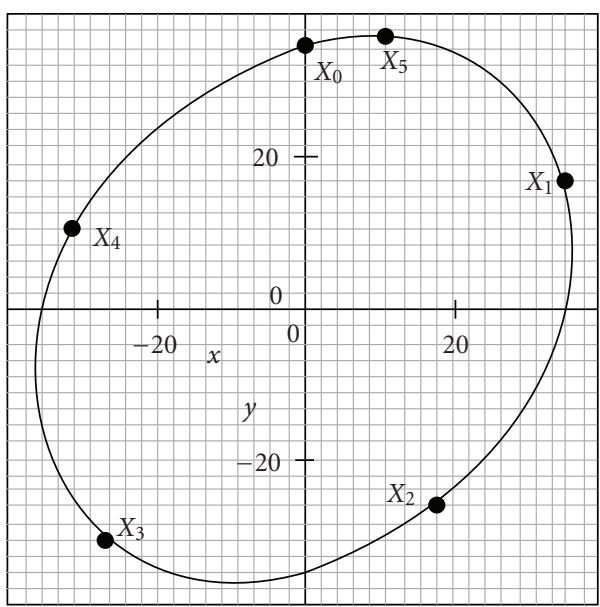

Figure 2.1. $a=1 / 2, X_{0}=(0,32)$.

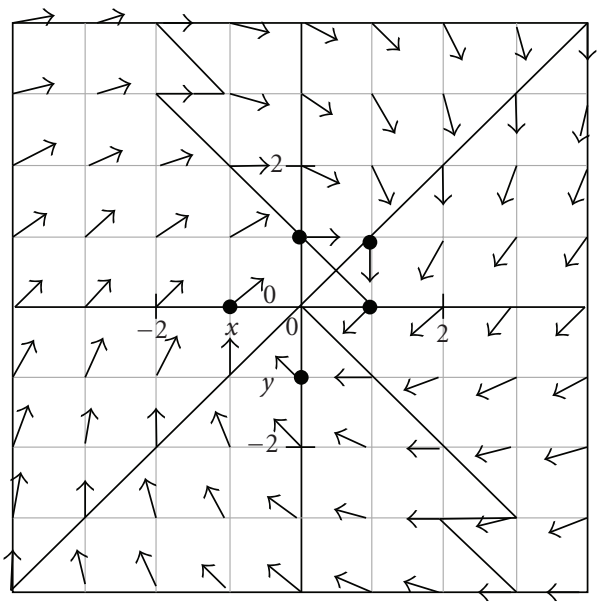

Figure 2.2. $a=1 / 3, X_{0}=(1,0)$.

THeOREM 2.2. For nonzero rational $a=p / q$ where $p$ and $q$ have no common factors, the number of distinct terms of a solution $\left(y_{n}\right)$ can be made arbitrarily large depending on the initial conditions.

Proof. Imitating the example of Figure 2.1, we choose initial conditions designed to deactivate the ceiling function for a finite number of terms, thereby turning the nonlinear equation (1.1) into a linear equation. Take $y_{0}=q^{m}, y_{1}=p q^{m-1}$ with arbitrarily large 


\section{Periodic solutions of arbitrary length}

positive $m$. As before, $A$ denotes the matrix underlying the linear system. By induction,

$$
A^{n}=\left(\begin{array}{cc}
\frac{-f_{n-2}}{q^{n-2}} & \frac{f_{n-1}}{q^{n-1}} \\
\frac{-f_{n-1}}{q^{n-1}} & \frac{f_{n}}{q^{n}}
\end{array}\right),
$$

where $f_{-1}=0, f_{0}=1$, and $f_{n}(p, q)=\sum_{k=0}^{\lfloor n / 2\rfloor}\left(\begin{array}{c}n-k \\ k\end{array}\right)(-1)^{k} p^{n-2 k} q^{2 k}$ for $n>0$. A consequence of taking $p$ and $q$ relatively prime is that $q$ never divides $f_{n}$ for $n \geq 0$; the coefficient of $p^{n}$ in $f_{n}$ is always 1 . Repeated application of $A$ to the initial vector $\left(0, q^{m}\right)$ gives the general form of the first $m+1$ iterates: $y_{n}=f_{n} q^{m-n}$. These are all distinct because the highest power of $q$ that divides each one is different.

In contrast, the following example shows that when $a$ is irrational, it does not follow that there are arbitrarily many distinct iterates simply because an initial condition is arbitrarily large (however, see Figure 4.1 below).

Example 2.3. Let $a=(\sqrt{5}-1) / 2=0.6180339 \ldots$, that is, $\theta=2 \pi / 5$ in (2.2), above. Let $y_{0}=1, y_{1}=10^{n}$ for $n \geq 0$. With this form of initial condition, all solutions have period 5 . Solutions are shown, below, for $n=0,1,2,3$, and 6 .

$\begin{array}{cccccccc}n=0 & 1, & 1, & 0, & -1, & 0, & 1, & 1, \ldots \\ 1 & 1, & 10, & 6, & -6, & -9, & 1, & 10, \ldots \\ 2 & 1, & 100, & 61, & -62, & -99, & 1, & 100, \ldots \\ 3 & 1, & 1000, & 618, & -618, & -999, & 1, & 1000, \ldots \\ 6 & 1, & 10^{6}, & 618033, & -618034, & 1-10^{6}, & 1, & 10^{6}, \ldots\end{array}$

The curious relation between $y_{2}$ and $y_{3}$ should be noted: sometimes $y_{3}=-y_{2}$ and sometimes $y_{3}=-y_{2}-1$. It is easy to see that $y_{2}=\left\lfloor 10^{n} a\right\rfloor$, where $\lfloor x\rfloor$ denotes the greatest integer not greater than $x$. With a little more work, using the fact that $a^{2}+a-1=0$, we can prove that $y_{3}=-y_{2}$ if and only if $1-a<\left\lceil 10^{n} a\right\rceil-10^{n} a$; otherwise, $y_{3}=-y_{2}-1$.

\section{Periodicity of solutions}

An involution is a map $V$ such that the square of $V$ is the identity, that is, $V^{2}=V \cdot V=I$ [2]. The following lemma provides basic machinery for proving that all solutions of (1.1) are periodic.

Lemma 3.1. Let $T$ be defined as in (2.1), above, and $S(x, y) \equiv T^{-1}(x, y)=(\lceil a x\rceil-y, x)$. The involution $V(x, y)=(y, x)$ satisfies $V T=S V$ and $T V=V S$. It follows that the mappings $V T, V S, T V$, and $S V$ are involutions.

Proof. We have

$$
V(T(x, y))=V(y,\lceil a y\rceil-x)=(\lceil a y\rceil-x, y)=S(y, x)=S(V(x, y))
$$


Multiplying $V T=S V$ on the left and right by $V$ yields $T V=V S$, which is used to prove that $V T V T=V V S T=I$. Thus, $V T$ is an involution and similarly so are $V S, T V$, and $S V$.

We call a solution of (1.1) invariant under $V$ if the point set $O=\left\{X_{n} \in \mathbb{Z}^{2}: X_{n}=\right.$ $\left.T^{n}\left(X_{0}\right), n=0,1,2, \ldots\right\}$ satisfies $V(O)=O$. Geometrically, for $V(x, y)=(y, x)$, this means that the plot of iterates is symmetric about the $45^{\circ}$ line, for example, see Figure 2.2, above. For rational $a$, numerical experiments have shown that this invariance is so prevalent, we conjecture it occurs with probability 1 . See the corollary to Theorem 3.2, below. For solutions invariant under $V$, the lemma establishes periodicity at once:

$$
X_{0}=T V T V\left(X_{0}\right)=T V T\left(X_{k}\right)=T V\left(X_{k+1}\right)=T\left(X_{m}\right)=X_{m+1} .
$$

The general periodicity result follows, with Figure 3.1, below, providing concrete support to the proof.

Theorem 3.2. For $a \in \mathbb{R},|a|<2$, all solutions of (1.1) are periodic.

Proof. Let a solution to (1.1) begin $y_{0}, y_{1}, \ldots$ Citing Remark 2.1, take $X_{0}=\left(\left\lceil a y_{0}\right\rceil-y_{1}, y_{0}\right)$ in $\mathbb{Z}^{2}$. The mappings $T, S$, and $V$ are defined as in (2.1) and Lemma 3.1, respectively. Set $Y_{0}=V\left(X_{0}\right)$ and $Y_{k(n)}=V\left(X_{n}\right)$ for $n=1,2, \ldots$ The value of $k$ is determined by use of the lemma in (3.3), below: $k$ is the number of times $S$ must be applied to the point $V\left(X_{n}\right)$ so that the points $Y_{k}, Y_{k-1}, Y_{k-2}, \ldots$ rotate (counterclockwise) back to $Y_{0}$. See Figure 3.1.

$$
\begin{aligned}
& Y_{k-1}=S\left(Y_{k}\right)=S V\left(X_{n}\right)=V T\left(X_{n}\right)=V\left(X_{n+1}\right), \\
& Y_{k+1}=T\left(Y_{k}\right)=T V\left(X_{n}\right)=V S\left(X_{n}\right)=V\left(X_{n-1}\right) .
\end{aligned}
$$

Again, by the lemma, (3.3) implies that $n$ applications of $T$ to $V\left(X_{n}\right)$ move $Y_{k}, Y_{k+1}$, $Y_{k+2}, \ldots$ (clockwise) to $Y_{k+n}=V\left(X_{0}\right)=Y_{0}$. Thus the sequence $\left(Y_{k}\right)$ is periodic. By definition, $\left(X_{n}\right)$ and $\left(Y_{k}\right)$ are in one-to-one correspondence by way of reflection across the $45^{\circ}$ line. Thus, $\left(X_{n}\right)$ is periodic. In particular, (3.3) implies $Y_{0}=V\left(X_{0}\right)=V\left(X_{n+k}\right)$; hence, $X_{0}=X_{n+k}$. In accordance with Remark 2.1, all solutions to (1.1) are periodic.

In Figure 3.1 the points of $\left(X_{n}\right)$

$$
(2,-3),(-3,-6),(-6,-5),(-5,-1),(-1,4),(4,7),(7,6),(6,2),(2,-3)
$$

are denoted by black circles. The points of $\left(Y_{k}\right)$, which are read from right to left in (3.4) with $V$ applied to each pair, are denoted by open circles in Figure 3.1. The following corollary deals with the special case where the initial pair lies on the $45^{\circ}$ line.

Corollary 3.3. For $a \in \mathbb{R},|a|<2$, and $X_{0}=\left(y_{0}, y_{0}\right)$ all solutions of (1.1) are invariant under $V$. 


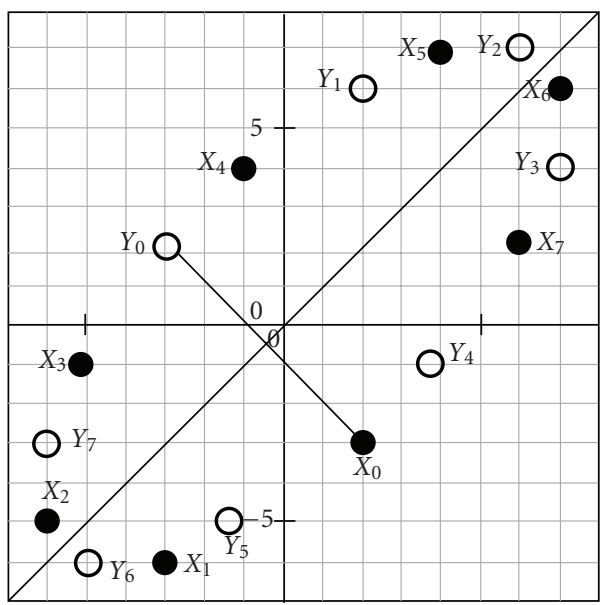

Figure 3.1. The subscripts of $X_{n}$ and its image under $V$ always sum to 8 .

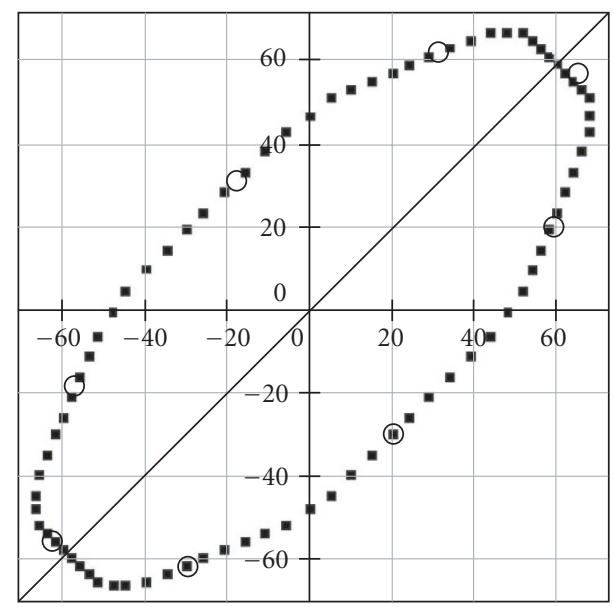

Figure 3.2. $X_{0}=(20,-30), a=7 / 5=1.4$ (black squares); $a=\sqrt{2}$ (open circles).

Proof. Solutions are periodic by Theorem 3.2. Suppose that, for a given $a$ and $X_{0}=\left(y_{0}\right.$, $\left.y_{0}\right)$, the resulting solution has smallest period $N$, so that $X_{0}=X_{N}$. Since $V(X)=X$ on the $45^{\circ}$ line, the lemma yields

$$
X_{1}=T\left(X_{0}\right)=T V\left(X_{0}\right)=V S\left(X_{N}\right)=V\left(X_{N-1}\right) .
$$




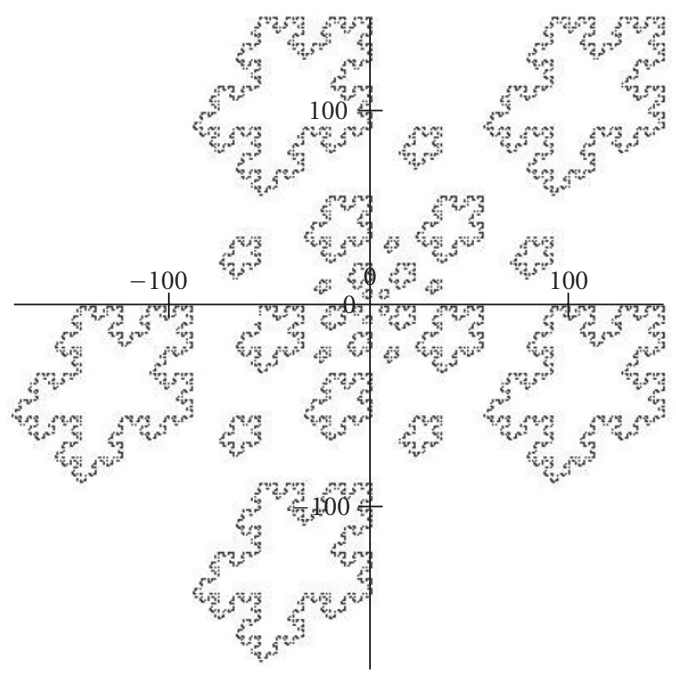

Figure 4.1. $a=(\sqrt{5}-1) / 2, \theta=2 \pi / 5$.

Next,

$$
X_{2}=T\left(X_{1}\right)=T V\left(X_{N-1}\right)=V S\left(X_{N-1}\right)=V\left(X_{N-2}\right) .
$$

Continuing in this way, $X_{k}=V\left(X_{N-k}\right)$ for $k=0,1,2, \ldots, N$.

By re-indexing, it is clear that if any iterate touches the $45^{\circ}$ line, the entire trajectory becomes symmetric about this line. Perhaps this explains why there are so many invariant solutions when $a$ is rational. The rotation angle $\theta=\arccos (a / 2)((2.2)$, above) is never a rational multiple of $\pi$ for nontrivial rational $a$, that is, $a \neq 0, \pm 1$ [3], indicating a large number of iterations relative to the size of $X_{0}$. The more densely packed with points a trajectory is, the greater the likelihood that one of them is located on the $45^{\circ}$ line. In any event, the small-period non-invariant solution with rational $a=7 / 5$ shown in Figure 3.1, above, was found by observing that $7 / 5=1.4$ is a fair approximation of $\sqrt{2}=2 \cos (2 \pi / 8)$ for a small initial point; hence, the period-8 solution (3.4). Predictably, with the same $a=7 / 5$ and larger $X_{0}=(20,-30)$, we get the period-79, $V$-invariant solution shown in Figure 3.2. In this solution, indicated by the dark squares, $X_{59}=(60,60)$. By the corollary, above, the entire orbit must line up with itself when reflected about the $45^{\circ}$ line. Maintaining $X_{0}=(20,-30)$ and changing $a$ to $\sqrt{2}$ gives back a period- 8 non-invariant solution shown by the open circles in Figure 3.2.

\section{Self-similarity and chaos}

The presence of symmetry in a brute iteration, perhaps just by accident of sheer numbers, is striking. Still more improbable is that, as the initial condition becomes larger, such 
8 Periodic solutions of arbitrary length

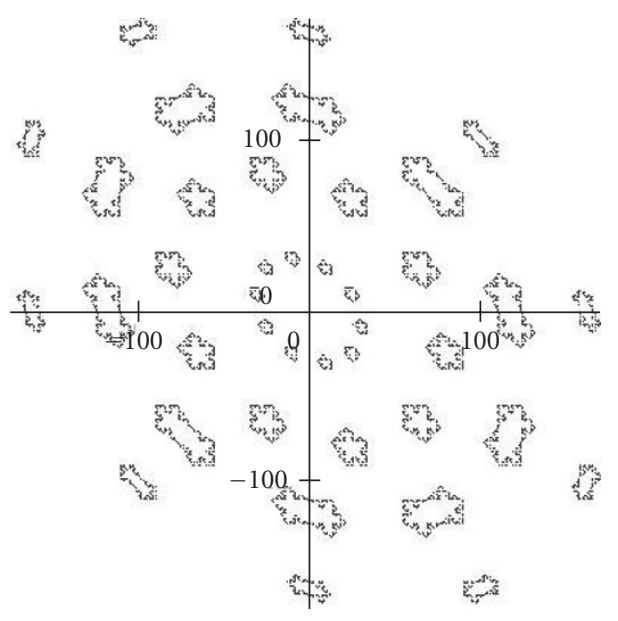

Figure 4.2. $a=(1-\sqrt{5}) / 2, \theta=3 \pi / 5$.

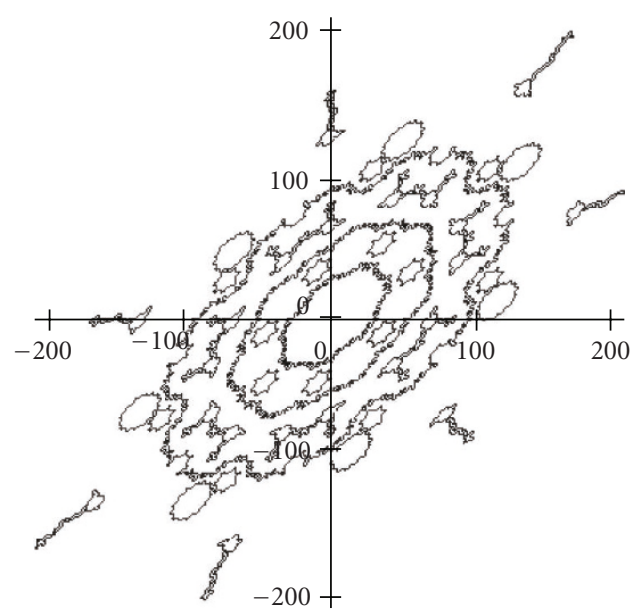

Figure 4.3. $a=2 \cos (2 \pi / 7), \theta=2 \pi / 7$.

a process can generate images with self-similar complexity as it winds blindly around the plane. See Figures 4.1 and 4.2. Each of Figures 4.1-4.4 shows a different choice of the parameter $a$ and several orbits for each choice. Even chaos is possible for specific initial conditions when $a=2 \cos (\theta)$ and $\theta$ is a rational multiple of $\pi$. Such solutions give rise to entirely unexpected structures as the initial point gets larger. For instance, Figure 4.4 shows just four orbits, with bizarre excrescences forming a single outermost orbit. Where it seems incontrovertible that the fractal stars in Figure 4.1 will continue to develop their repetitive complexity, there is no telling what may emerge from the vaguely 


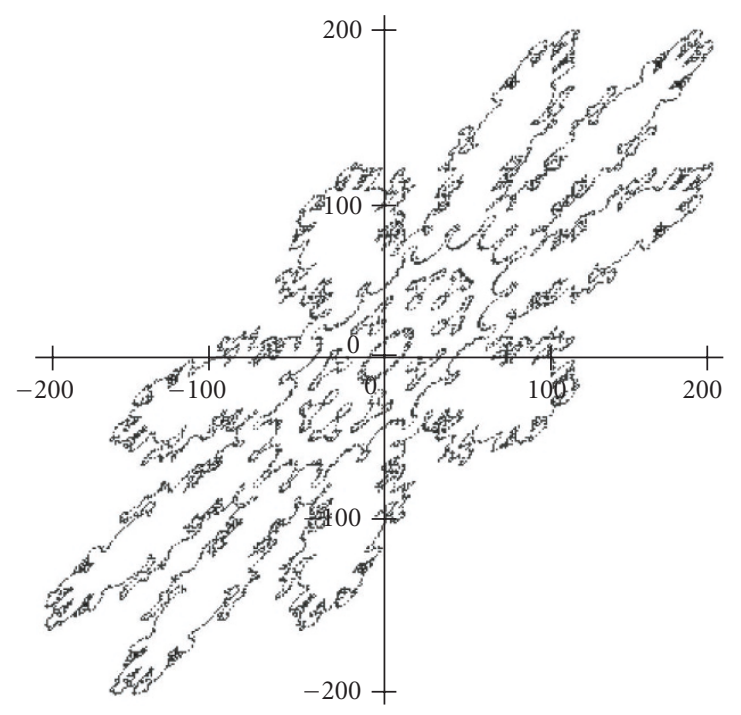

Figure 4.4. $a=2 \cos (2 \pi / 9), \theta=2 \pi / 9$.

bio-reproductive shapes in Figure 4.3 as we zoom out. Evidently, only the distance from the origin of a properly chosen initial pair limits the complexity of these images.

\section{References}

[1] D. Clark and J. T. Lewis, Symmetric solutions to a Collatz-like system of difference equations, Congr. Numer. 131 (1998), 101-114.

[2] G. James and R. C. James, Mathematics Dictionary, 4th ed., Van Nostrand Reinhold, New York, 1976.

[3] I. Niven, Irrational Numbers, The Carus Mathematical Monographs, no. 11, The Mathematical Association of America. Distributed by John Wiley \& Sons, New York, 1956.

Dean Clark: University of Rhode Island, Kingston, RI 02881, USA

E-mail address:dclark@uri.edu 